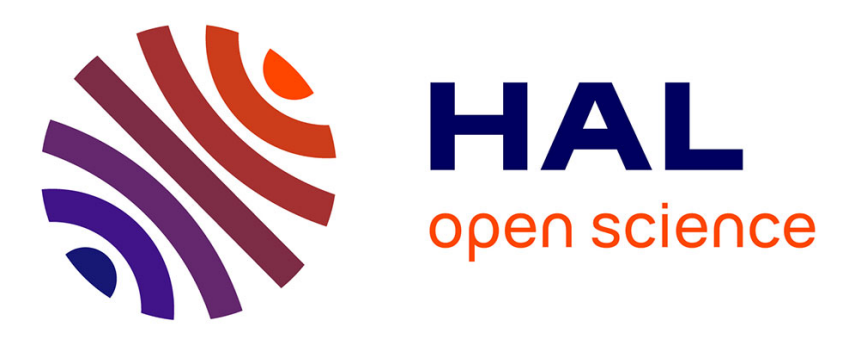

\title{
Image-based Control of Mobile Robot with Central Catadioptric Cameras
}

Hicham Hadj Abdelkader, Y. Mezouar, Nicolas Andreff, Philippe Martinet

\section{To cite this version:}

Hicham Hadj Abdelkader, Y. Mezouar, Nicolas Andreff, Philippe Martinet. Image-based Control of Mobile Robot with Central Catadioptric Cameras. 2005 IEEE International Conference on Robotics and Automation, Apr 2005, Barcelona, Spain. pp.3522-3527, 10.1109/ROBOT.2005.1570655 . hal02467141

\section{HAL Id: hal-02467141 \\ https://hal.inria.fr/hal-02467141}

Submitted on 5 Feb 2020

HAL is a multi-disciplinary open access archive for the deposit and dissemination of scientific research documents, whether they are published or not. The documents may come from teaching and research institutions in France or abroad, or from public or private research centers.
L'archive ouverte pluridisciplinaire $\mathbf{H A L}$, est destinée au dépôt et à la diffusion de documents scientifiques de niveau recherche, publiés ou non, émanant des établissements d'enseignement et de recherche français ou étrangers, des laboratoires publics ou privés. 


\title{
Image-based Control of Mobile Robot with Central Catadioptric Cameras
}

\author{
Hicham Hadj Abdelkader, Youcef Mezouar, Nicolas Andreff and Philippe Martinet \\ LASMEA, 24 avenue des Landais, 63177 AUBIERE-FRANCE \\ hadj,mezouar,andreff,martinet@lasmea.univ-bpclermont.fr
}

\begin{abstract}
To close the loop between motion and vision, tracked visual features must remain in the camera field of view (visibility constraint). To overcome the visibility constraint, visual servoing methods can benefit from panoramic sensors such as catadioptric cameras (combining both mirrors and lenses). In this paper, we present a vision-based framework to control a nonholonomic mobile robot using a catadioptric imaging system. We particularly focus on a suitable catadioptric image-based control strategy of a nonholonomic robot in order to follow a 3D straight line. Such strategy can be applied to navigate in indoor or urban environment since the extraction and the tracking of straight lines are natural. First the control objectives are formulated in the catadioptric image space. The control law is then designed according to a well suited chained system for a mobile robot state vector directly expressed in the image space using a generic camera model. Simulation results illustrate the control strategy in the case of hypercatadioptric and paracatadioptric cameras.
\end{abstract}

Index Terms-Omnidirectional vision, mobile robot control, visual servoing.

\section{INTRODUCTION}

In the last years, the use of visual observations to control robot motions has been extensively studied. Indeed, computer vision can provide a robotic system with the ability to operate in circumstances and environments which can not be accurately controlled. This approach is referred to in the literature as visual servoing. Visual servoing schemes are traditionally classified in three groups: position-based[8], image-based [11] and hybrid-based control [15]. The first one, based on the computation of a 3-D Cartesian error, requires generally a perfect $\mathrm{CAD}$ model of the object and a calibrated camera to obtain unbiased pose estimation. In the second approach, the control loop is directly closed in the image space and thus the full model of the object is not to be known accurately. Hybrid-based visual servoing methods combine image and 3-D informations to design the input vector. In this paper, we will focus on image based methods.

Conventional cameras suffer from restricted field of view. Ambiguities and confusion between translation and rotation may arise whenever the translation direction lies outside the camera field of view. Omnidirectional cameras overcome this problem since they provide a 360 degrees field of view of the surroundings. Many applications in vision-based robotics, such as mobile robot localisation [5] and navigation [25], can benefit from panoramic field of view provided by omnidirectional cameras. Visual servoing applications can also benefit from cameras with a wide field of view. Indeed, classical visual servoing techniques make assumptions on the link between the initial, current and desired images. They require correspondences between the visual features extracted from the initial image with those obtained from the desired one. These features are then tracked during the camera (and/or the object) motion. If these steps fail, the visually based robotic task can not be achieved [6]. Typical cases of failure arise when matching joint images features is impossible (for example when no joint features belongs to initial and desired images) or when some parts of the visual features get out of the field of view during the servoing. Some methods have been proposed to resolve this deficiency based on path planning [16], switching control [7], zoom adjustment [18] or geometrical and topological considerations [21]. However, such strategies are sometimes delicate to adapt to a generic setup. Omnidirectional cameras naturally overcome this problem. As a consequence, such sensors have been successfully integrated as part of a closed loop feedback control system [24], [3].

In the literature, there have been several methods proposed to increase the field of view of cameras systems [4]. One effective way is to combine mirrors with conventional imaging system. The obtained sensors are referred to as catadioptric imaging systems. The resulting imaging systems have been termed central catadioptric when a single projection center describes the world-image mapping. From a practical view point, a single center of projection is a desirable property for an imaging system [1]. Baker and Nayar in [1] derive the entire class of catadioptric systems with a single viewpoint.

Image-based visual servoing methods have been firstly developed for manipulators. In [22], Tsakiris et al point out that image-based visual servoing techniques can be extended to nonholonomic mobile robots by adding degrees of freedom to the hand-eye system. This paper proposes to embed the visual servoing control scheme in the task function formalism [8]. Vision-based mobile robotic tasks such as wall following or self positioning with respect to landmarks is thus possible using this framework [12]. Without these extra degrees of freedom, the pose of the camera with respect to the target can not be stabilized involving only a state feedback. However, it is possible to exploit work which aims to control a nonholonomic wheeled mobile robot moving on a plane [13] in order 
to track a non-timed analytical path in the image space without recovering any 3D parameters of the path. Yi Ma et al in [14] propose a theoretical framework to track a ground curve by approximating its projection in the image plane of a conventional camera with piecewise analytic curves with linear curvature. Usher et al [23] the authors propose a switching controller to regulate the pose of a vehicle using information provided by an omnidirectional camera. In [24], the problem of formation control is addressed by specifying the desired formation in the image plane of an omnidirectional camera. The global control problem is translated into separate visual servoing tasks for each follower.

In this paper, we particularly focus on a suitable catadioptric image-based control strategy of a nonholonomic robot in order to follow a 3D straight line. Such a strategy can be applied to navigation in indoor or urban environment since the extraction and the tracking of straight lines are natural. The first contribution is to formulate the control objectives in the catadioptric image space. The second one is to tightly couple catadioptric visual servoing and mobile robot control. Indeed, the control law is designed according to a well suited chained system with a state vector directly expressed in the image space.

The remainder of this paper is organized as follows. In Section II, following the description of the catadioptric camera model, lines projections in the image plane is studied. In Section III, the problem we address is described and the models used through the paper are introduced. Section IV is devoted to the control law design. In Section $\mathrm{V}$, simulated results are presented.

\section{CAMERA MODEL AND IMAGE FORMATION OF LINES}

In this section, we describe the projection model for catadioptric cameras and then we focus on 3D lines features.

\section{A. Catadioptric camera model}

As noted in the introduction, a single center of projection is a desirable property for an imaging system. A single center implies that all lines passing through a 3D point and its projection in the image plane pass through a single point in $3 \mathrm{D}$ space. Conventional perspective cameras are single view point sensors with limited field of view (typically 45 degrees). As shown in [1], a central catadioptric system can be built by combining an hyperbolic, elliptical or planar mirror with a perspective camera (see Fig. 1) and a parabolic mirror with an orthographic camera.

Let $\mathcal{F}_{c}$ and $\mathcal{F}_{m}$ be the frames attached to the conventional camera and to the mirror respectively (see Fig. 1). In the sequel, we suppose that $\mathcal{F}_{c}$ and $\mathcal{F}_{m}$ are related by a translation along the $\mathrm{Z}$-axis. The centers $C$ and $M$ of $\mathcal{F}_{c}$ and $\mathcal{F}_{m}$ will be termed optical center and principal projection center respectively. Let $\mathcal{X}$ be a 3D point with coordinates $\mathbf{X}=\left[\begin{array}{lll}X & Y & Z\end{array}\right]^{T}$ with respect to $\mathcal{F}_{m}$. According to the generic projection model [10], $\mathcal{X}$ is projected in the image plane to a point $\mathbf{x}=\left[\begin{array}{lll}x & y & 1\end{array}\right]^{T}$ with:

$$
\mathbf{x}=\mathbf{K f}(\mathbf{X})
$$

where $\mathbf{K}$ denotes the triangular calibration matrix of the catadioptric camera, containing mirror and lens intrinsic parameters and:

$$
\mathbf{f}(\mathbf{X})=\left(\begin{array}{c}
\frac{X}{Z+\xi \sqrt{X^{2}+Y^{2}+Z^{2}}} \\
\frac{Y}{Z+\xi \sqrt{X^{2}+Y^{2}+Z^{2}}} \\
1
\end{array}\right)
$$

where $\xi$ is a mirror intrinsic parameter.

In the sequel, we will assume that the $\mathbf{K}$-matrix is the identity (calibrated configuration [26]), the mapping function describing central catadioptric projection is then given by: $\mathbf{x}=\mathbf{f}(\mathbf{X})$.

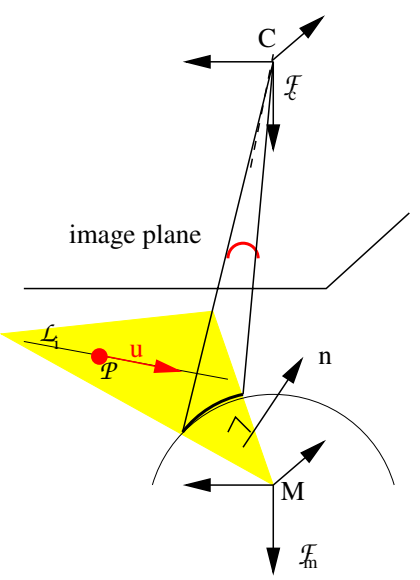

Fig. 1. Projection of a 3D line onto a conic in the image plane

\section{B. Catadioptric projection of Lines}

Let $\mathcal{P}$ be a $3 \mathrm{D}$ point and $\mathbf{u}=\left(u_{x}, u_{y}, u_{z}\right)^{T}$ a unit vector expressed in the mirror frame and $\mathcal{L}$ the $3-\mathrm{D}$ line they define (refer to Fig. 1). Define $\mathbf{n}=\overrightarrow{M \mathcal{P}} \times \mathbf{u}=$ $\left(n_{x}, n_{y}, n_{z}\right)^{T}$ and remark that this vector is independent of the point we choose on the line. Thus the Euclidean Plücker coordinates are defined as $\mathcal{L}:\left(\begin{array}{cc}\mathbf{n}^{T} & \mathbf{u}^{T}\end{array}\right)^{T}$ with $\mathbf{n}^{T} \mathbf{u}=0$. The $\mathbf{n}$-vector is orthogonal to the interpretation plane $\Pi$ defined by the line and the principal projection center:

$$
\mathbf{X}=[X, Y, Z]^{T} \in \Pi \Longleftrightarrow n_{x} X+n_{y} Y+n_{z} Z=0
$$

Let $\mathcal{S}$ be the intersection between the interpretation plane and the mirror surface. $\mathcal{S}$ represents the line projection in the mirror surface. Note that all 3D lines of $\Pi$ are projected onto $\mathcal{S}$. The projection $\mathcal{S}$ of $\mathcal{L}$ in the catadioptric image plane is then obtained using a conventional imaging system. It can be shown using (1) and (2) (or following [17]) that $3 \mathrm{D}$ points lying on $\mathcal{L}$ are mapped into points in the image $\mathrm{x}$ which verify:

$$
\mathbf{x}^{T} \Omega \mathbf{x}=0
$$

with :

$$
\boldsymbol{\Omega}=\left(\begin{array}{ccc}
n_{x}^{2}\left(1-\xi^{2}\right)-n_{z}^{\eta} \xi^{2} & n_{x} n_{y}\left(1-\xi^{2}\right) & (2 \eta-3) n_{x} n_{z}^{\eta-1} \\
n_{x} n_{y}\left(1-\xi^{2}\right) & n_{y}^{2}\left(1-\xi^{2}\right)-n_{z}^{\eta} \xi^{2} & (2 \eta-3) n_{y} n_{z}^{\eta-1} \\
(2 \eta-3) n_{x} n_{z}^{\eta-1} & (2 \eta-3) n_{y} n_{z}^{\eta-1} & n_{z}^{\eta}
\end{array}\right)
$$




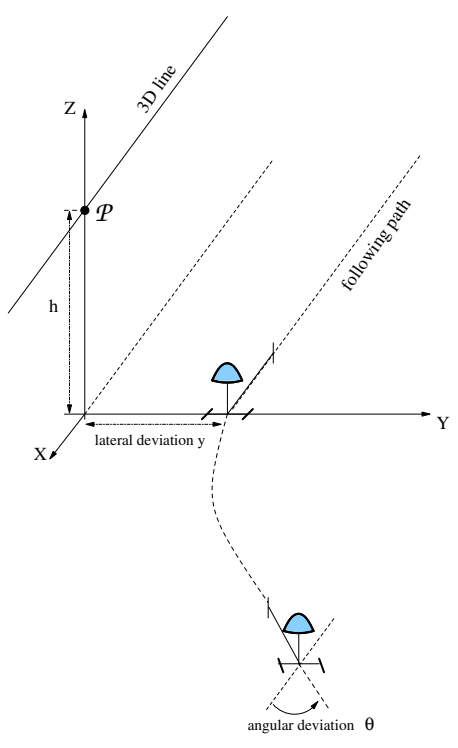

Fig. 2. The task to be achieved

where $\eta=2$ in the general case and $\eta=1$ for the combination parabolic mirror-orthographic camera. Note that the projection of a line is fully defined by the vector $\mathbf{n}$. A line in space is thus mapped onto the image plane to a conic curve. The relation (3) defines a quadratic equation:

$$
A_{0} x^{2}+A_{1} y^{2}+2 A_{2} x y+2 A_{3} x+2 A_{4} y+A_{5}=0
$$

with:

$$
\left\{\begin{array}{l}
A_{0}=s\left(n_{x}^{2}\left(1-\xi^{2}\right)-n_{z}^{\eta} \xi^{2}\right) \\
A_{1}=s\left(n_{y}^{2}\left(1-\xi^{2}\right)-n_{z}^{\eta} \xi^{2}\right) \\
A_{2}=s n_{x} n_{y}\left(1-\xi^{2}\right) \\
A_{3}=s(2 \eta-3) n_{x} n_{z}^{\eta-1} \\
A_{4}=s(2 \eta-3) n_{y} n_{z}^{\eta-1} \\
A_{5}=s n_{z}^{\eta}
\end{array}\right.
$$

Let us note that the equation (4) is defined up to a scale factor $s$. We thus normalize (4) using $A_{5}$ to obtain unambiguous representations. The quadratic equation is thus rewritten as follows:

$$
B_{0} x^{2}+B_{1} y^{2}+2 B_{2} x y+2 B_{3} x+2 B_{4} y+1=0
$$

with $B_{i}=\frac{A_{i}}{A_{5}}$. The case $n_{z}=0$ corresponds to a degenerate configuration of our representation where the optical axis lies on the interpretation plane. In this case, the projection of the $3 \mathrm{D}$ line in the image can be shown to be a line passing through the image center. In the following, we will only consider the non degenerated case.

\section{PROBLEM FORMULATION}

Throughout the paper, we consider a nonholonomic system with car-like kinematics where the embedded catadioptric system looks at the upwards from the ground. In the first part of this section, the control objective is presented. In the second part, the control objective is reformulated in the catadioptric image space.

\section{A. Control objective in the robot workspace}

Let $\mathcal{F}_{r}$ be the frame attached to the robot control frame. It is supposed confounded with $\mathcal{F}_{m}$. In this configuration, the camera optical axis coincides with the rotation axis of the mobile robot and the camera optical center is confounded with the axle midpoint of the mobile robot. The camera frame and the mobile robot are thus subjected to the same kinematic constraints. The kinematic screw is only composed with a linear velocity along the $X$-axis of the camera frame and an angular velocity about the optical axis. This is still true if the optical center is not confounded with the axle midpoint but lies on the Z-axis of $\mathcal{F}_{m}$.

Consider a 3D straight line $\mathcal{L}$ parallel to the $X Y$-plane of $\mathcal{F}_{r}$ and parallel to the $X$-axis of the world frame. It is projected onto the image plane according to (3). The control objective is to drive the $X$-axis of the control frame parallel to the line while keeping a constant distance to the line (see Fig. 2). The state of the mobile robot can be described by the vector $X_{r}=\left[\begin{array}{lll}x & y & \theta\end{array}\right]^{T}$, where $x$ and $y$ are the coordinates of the camera frame center with respect to the world frame and $\theta$ is the angular deviation with respect to the straight line (see Fig. 3). The task is achieved when the lateral deviation $y$ is equal to the desired one $y^{*}$ and the angular deviation $\theta$ is null. Thanks to the properties of chained system, we are able to decouple the lateral control from the longitudinal deviation if $v \neq 0$. The state vector $X_{r}$ can thus be reduced to $\left[\begin{array}{ll}y & \theta\end{array}\right]^{T}$. We now describe how to translate the control objective in the catadioptric image space.

\section{B. Formulation of the control objective in the image space}

The projection of a 3-D line $\mathcal{L}$ in the catadioptric image is fully defined by the normal vector $\mathbf{n}$ to the interpretation plane (refer to Section II). The direction of $\mathcal{L}$ is given by the unit vector $\mathbf{u}$ of coordinates with respect to the control frame given by:

$$
\mathbf{u}=\left(\begin{array}{c}
\cos \theta \\
-\sin \theta \\
0
\end{array}\right)
$$

The previous relation defines the vector $\mathbf{u}$ as a function of the angular deviation $\theta$ (see Fig. 3). It is thus independent of the lateral deviation $y$ of the mobile robot with respect to the line. Since $\mathbf{n}=\overrightarrow{M \mathcal{P}} \times \mathbf{u}=\left[\begin{array}{lll}n_{x} & n_{y} & n_{z}\end{array}\right]^{T}$ is independent of the point $\mathcal{P}$ we choose on the line, $\mathcal{P}$ can be taken as the point of coordinates $\mathcal{P}=\left[\begin{array}{lll}y \sin \theta & y \cos \theta & h\end{array}\right]^{T}$ with respect to the control frame ( $h$ denotes the height of the line from the ground). The normal vector to the interpretation plane $\mathbf{n}$ is thus given by:

$$
\mathbf{n}=\left(\begin{array}{c}
-h \sin \theta \\
-h \cos \theta \\
y
\end{array}\right)
$$

Note that when the $X$-axis of the control frame is parallel to the line $\mathcal{L}$ (i.e when the angular deviation is null) only its last component varies as the longitudinal deviation. Conversely, the two first components of $\mathbf{n}$ depend only 


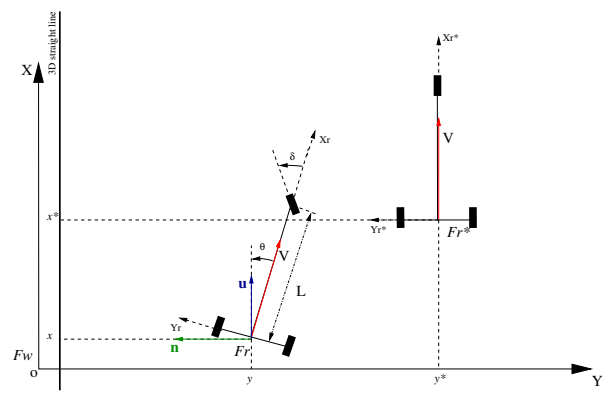

Fig. 3. Modelling the car-like vehicle

of the angular deviation. Let us now represent the angular and longitudinal deviation as functions of image features.

Consider the observation vector $\mathbf{s}=\left[\begin{array}{ll}B_{3} & B_{4}\end{array}\right]^{T}$ extracted from the projection of line $\mathcal{L}$ in the catadioptric image $\left(B_{3}\right.$ and $B_{4}$ has been defined in Section II). The observation vector $\mathbf{s}$ fully represents the line projection and it is minimal. An important remark is that the observation vector $\mathbf{s}$ is the perspective projection of the normal vector $\mathbf{n}$. Knowing that:

$$
\left\{\begin{array}{l}
B_{3}=\beta \frac{n_{x}}{n_{z}} \\
B_{4}=\beta \frac{n_{y}}{n_{z}}
\end{array}\right.
$$

where $\beta=2 \eta-3$ (i.e. $\eta=2$ in the general case and $\eta=1$ for the combination of parabolic mirror with orthographic camera), we get:

$$
y=\frac{h}{\sqrt{B_{3}^{2}+B_{4}^{2}}}
$$

$B_{3}^{2}+B_{4}^{2}$ is null only if the 3D line lies on the $X Y$ plane of the camera frame $\mathcal{F}_{m}$ (i.e. the center of the line projection in the catadioptric image is confounded with the optical center). The angular deviation can easily be rewritten as a function of the observation vector by combining equations (7) and (8): $\tan \theta=\frac{B_{3}}{B_{4}}$ and thus:

$$
\theta=\tan ^{-1} \frac{B_{3}}{B_{4}}
$$

$B_{4}$ is null only if the $X$-axis of the mirror frame is perpendicular to the 3D line (i.e $\theta=\frac{\pi}{2}[\pi]$ ).

The reduced state vector of the mobile robot $[y \theta]$ can thus be expressed directly in the sensor space according to (9) and (10).

\section{Control LaW DESIGN}

The control objective is to drive the $X$-axis of the control frame parallel to the line while keeping a constant distance to the line (see Fig. 2). The task is achieved when the lateral deviation $y^{\prime}$ is equal to the desired one $y^{\prime *}$, (without loss of generality, we define the lateral deviation by $y=$ $\left.y^{\prime}-y^{\prime *}\right)$ and the angular deviation $\theta$ is null. To achieve this control objective, chained systems properties are very interesting. A chained system results from a conversion of a non linear model into an almost linear one [19] and [20]. As long as the robot longitudinal velocity $v$ is non zero, the performances of a path tracking can be determined in terms of settling distance. We consider a nonholonomic system with car-like kinematics. The car-like vehicle is supposed to move on a perfect horizontal ground under the conditions of pure rolling and non-slipping. The control vector is $\mathbf{u}_{\mathbf{c}}=$ $\left[\begin{array}{ll}v & \delta\end{array}\right]^{T}$. The state and control vectors are related by the following kinematics equations:

$$
\left\{\begin{array}{l}
\dot{x}=v \cos \theta \\
\dot{y}=v \sin \theta \\
\dot{\theta}=\frac{v}{L} \tan \delta
\end{array}\right.
$$

Note that the previous kinematics equations can be translated in the image space using the so called interaction matrix or image Jacobian [11]. In order to design the control law as simply as possible, the kinematics equations in Cartesian space will be exploited and the equations (9) and (10) will be used to express the control law in the image space.

Let us now convert the state space model (11) into a 3 dimensional chained system $\left[\begin{array}{lll}a_{1} & a_{2} & a_{3}\end{array}\right]^{T}$ with a two dimensional control vector $\left[\begin{array}{ll}m_{1} & m_{2}\end{array}\right]^{T}$. The derivative of such a chained form with respect to time is:

$$
\left\{\begin{array}{l}
\dot{a_{1}}=m_{1} \\
\dot{a_{2}}=a_{3} m_{1} \\
\dot{a_{3}}=m_{2}
\end{array}\right.
$$

In order to point out that a chained system is almost linear, it is derivated with respect to the state variable $a_{1}$ :

$$
\left\{\begin{array}{l}
a_{1}^{\prime}=1 \\
a_{2}^{\prime}=a_{3} \\
a_{3}^{\prime}=m_{3}
\end{array}\right.
$$

where $m_{3}=\frac{m_{2}}{m_{1}}$. Choosing $a_{1}=x$, the linear system (11) is independent from the longitudinal velocity $v$ since it is driven by a variable homogeneous to the distance covered by the mobile robot. The input variable $m_{1}$ is thus given by (see equations (11) and (12)): $m_{1}=\dot{x}=v \cos \theta$. Let us now define $a_{2}=y$. Then it comes: $\dot{a_{2}}=v \sin \theta=a_{3} m_{1}$. Therefore $a_{3}$ must be chosen as:

$$
a_{3}=\frac{\dot{a_{2}}}{m_{1}}=\tan \theta
$$

Consequently, $a_{3}$ is not defined for $\theta=\frac{\pi}{2}[\pi]$. The control $m_{2}$ can be deduced from (12):

$$
m_{2}=\frac{v \tan \delta}{L \cos ^{2} \theta}
$$

Since the state space model (13) is linear, a natural and simple expression for the control law is:

$$
m_{3}=-K_{d} a_{3}-K_{p} a_{2} \quad\left(K p, K_{d}\right) \in \mathcal{R}^{2}
$$

Reporting (14) in (13) leads to: $a_{2}^{\prime}+K_{d} a_{2}^{\prime}+K_{p} a_{2}=0$.

This last equation implies that both $a_{2}$ and $a_{3}$ converge to zero, independently of the longitudinal velocity of the vehicle as long as $v \neq 0$. Since $a_{2}=y$ and $a_{3}=\tan \theta$, the same conclusion holds for $y$ and $\theta$. The performance of the proportional-derivative controller and the settling distance for the regulation of $y$ are determined by the gains $K_{p}$ 
and $K_{d}$. Moreover by combining the following relations $a_{2}=y, a_{3}=\tan \theta$ and (14), we get:

$$
\delta=\tan ^{-1}\left[L \cos ^{3} \theta\left(-K_{d} \tan \theta-K_{p} y\right)\right]
$$

According to (9) and (10), the control law (15) can be rewritten as:

$\delta=\tan ^{-1}\left[L \cos ^{3}\left(\tan ^{-1} \frac{B_{3}}{B_{4}}\right)\left(-K_{d} \frac{B_{3}}{B_{4}}-K_{p} \frac{h}{\sqrt{B_{3}^{2}+B_{4}^{2}}}\right)\right]$

The previous equation presents the control law as a function of the image features $B_{3}, B_{4}$ and the constant parameters $h$. In a real setup, $h$ is estimated and taken as $\widehat{h}=h \cdot \Delta h$. However, the last part of the control law (16) can be written as $K_{p}^{\prime} \frac{h}{\sqrt{B_{3}^{2}+B_{4}^{2}}}$ with $K_{p}^{\prime}=K_{p} \Delta h$, this means that a bad estimation of $h$ acts as a factor on the gain $K_{p}$ and thus modifies the control law performances. In practice, $\Delta h$ is over-estimated to tune the gains.

\section{Simulation RESUlts}

In this section, we present simulation results of central catadioptric vision-based control of a mobile robot using the control law (16). In the first simulation, a paracatadioptric system (a parabolic mirror combined with an orthographic lens) is used, and in the last one, an hypercatadioptric system (an hyperbolic mirror combined with a perspective lens) is considered. We used a car-like vehicle kinematics as model (refer to equation (11)). The gains were set to $\left(K_{p}, K_{d}\right)=(1,2)$ for these simulations. The initial and desired states of the mobile robot with respect to the 3D line are the same in these simulations. Figure 4 shows the initial spatial configurations of the line and the camera (or mobile robot). To be close to a real setup, an estimated calibration matrix $\widehat{\mathbf{K}}$ (with an error of $\pm 10 \%$ on the focal length and \pm 5 pixels on the coordinates of the image center) is used. $\widehat{h}$ has been set to $1.2 \mathrm{~m}$ whereas the real value is $1 \mathrm{~m}$, and an image noise has been added when extracting the observation vector $\mathbf{s}$ (maximum amplitude of \pm 5 pixels) in the case of a paracatadioptric camera. Unfortunately, when using an hypercatadioptric camera, robust algorithm is necessary to extract a portion of conics. For that reason, noise has been added directly to the normal vector $\mathbf{n}$.

The state of the mobile robot is derived from features extracted from the catadioptric image of a line. In the case of paracatadioptric cameras, the image of a line is a portion of circle. The center of this circle is given by the perspective projection of the normal vector $\mathbf{n}$. This is not true in the case of hypercatadioptric cameras. The image of a line becomes a portion of a conic.

As shown in Figures 6 and 8, angular and lateral deviations are well regulated to zero in both cases (paracatadioptric and hypercatadioptric cameras). Note also that these deviations are similar in the case of a paracatadioptric camera or an hypercatadioptric camera. The projection of the line in the paracatadioptric (resp. hypercatadioptric) image at the initial position of the mobile robots is shown in
Figure 5 with blue color (resp. Figure 7). It joins its image at the desired location given in Figure 5 with red color (resp. Figure 7) when the task is achieved. The trajectory of the line projection in the image is shown in Figure 5 with green color (resp. Figure 7) and confirm that the task is correctly realized.

Note finally that fitting a conics to image measurements is not a simple issue. Such process is sensitive to noise measurements and unstable. Moreover, one will only see a portion of the conic due to occlusions and camera motions. This is particulary true when an hypercatadioptric camera is used since the projection of a $3 \mathrm{D}$ line can potentially be any conics. We are currently working on this issue. Fortunately, the projection of 3D lines onto the image plane of a paracatadioptric camera are circles. In this case, stable and robust algorithms can be used (see [2] and [9]).

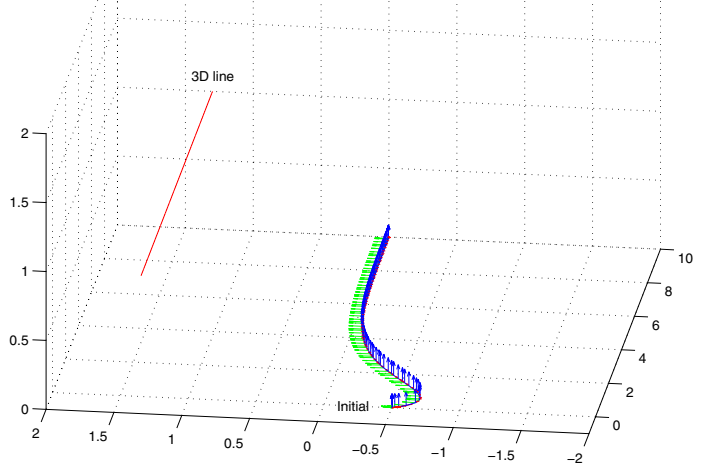

Fig. 4. Line configuration and robot trajectory

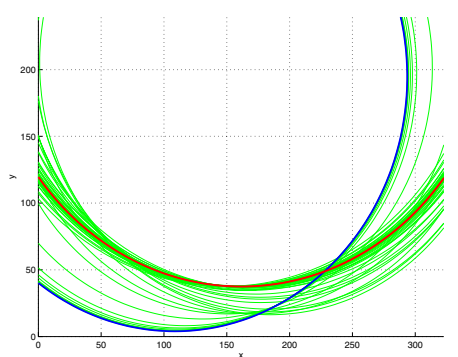

Fig. 5. Trajectories in the image plane of line projection with a paracatadioptric camera

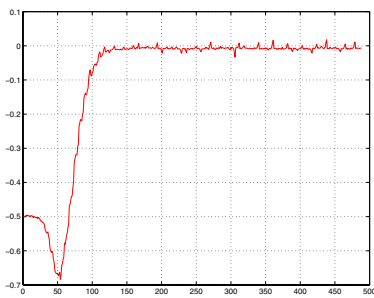

Lateral deviation $[\mathrm{m}]$

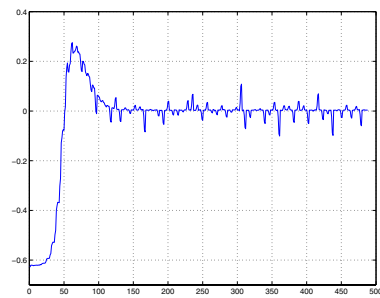

Angular deviation [rad]
Fig. 6. Simulation with a paracatadioptric camera 


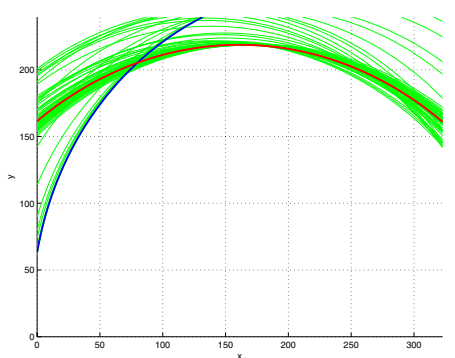

Fig. 7. Trajectories in the image plane of line projection with an hypercatadioptric camera

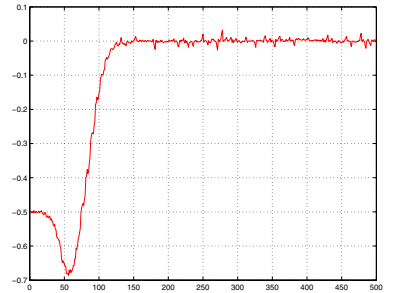

Lateral deviation $[\mathrm{m}]$

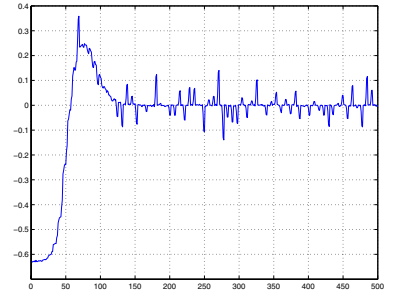

Angular deviation [rad]
Fig. 8. Simulation with an hypercatadioptric camera

\section{CONCLUSIONS}

Visibility constraints are extremely important for visual servoing applications. To overcome these constraints, the wide field of view of central catadioptric cameras can be exploited. We have addressed the problem of controlling the motion of a nonholonomic mobile robot directly in the image space, by incorporating observations from a catadioptric camera to follow a 3D straight line. We have detailed the establishment of a control law based on a chained form for a state vector directly expressed in the image space. The proposed approach can be used with all central cameras (including the conventional ones). It has been validated in simulation with paracatadioptric and hypercatadioptric cameras. The simulations show that the control law is robust with respect to noise measurements and modelling errors. Future work will be devoted to study the case of general path following using central catadioptric cameras, and to validate it in a real experimental setup.

\section{REFERENCES}

[1] S. Baker and S. K. Nayar. A theory of single-viewpoint catadioptric image formation. International Journal of Computer Vision, 35(2):1-22, November 1999.

[2] J. Barreto and H. Araujo. Direct least square fitting of paracatadioptric line images. In OMNIVIS 2003 - Workshop on Omnidirectional Vision and Camera Networks, pages 78-83, Madison, Wisconsin, USA, June 2003.

[3] J. P. Barreto, F. Martin, and R. Horaud. Visual servoing/tracking using central catadioptric images. In ISER2002 - 8th International Symposium on Experimental Robotics, pages 863-869, Bombay, India, July 2002.

[4] R. Benosman and S. Kang. Panoramic Vision. Springer Verlag ISBN 0-387-95111-3, 2000.

[5] P. Blaer and P.K. Allen. Topological mobile robot localization using fast vision techniques. In IEEE International Conference on Robotics and Automation, pages 1031-1036, Washington, USA, May 2002.
[6] F. Chaumette. Potential problems of stability and convergence in image-based and position-based visual servoing. The Confluence of Vision and Control, D. Kriegman, G. Hager, A. Morse (eds), LNCIS Series, Springer Verlag, 237:66-78, 1998.

[7] G. Chesi, K. Hashimoto, D. Prattichizzo, and A. Vicino. A switching control law for keeping features in the field of view in eye-in-hand visual servoing. In IEEE International Conference on Robotics and Automation, pages 3929-3934, Taipei, Taiwan, September 2003.

[8] B. Espiau, F. Chaumette, and P. Rives. A new approach to visual servoing in robotics. IEEE Transactions on Robotics and Automation, 8(3):313-326, June 1992.

[9] M. Fitzgibbon, A. W.and Pilu and R. B. Fisher. Direct least-squares fitting of ellipses. 21(5):476-480, May 1999.

[10] C. Geyer and K. Daniilidis. A unifying theory for central panoramic systems and practical implications. In European Conference on Computer Vision, volume 29, pages 159-179, Dublin, Ireland, May 2000.

[11] S. Hutchinson, G.D. Hager, and P.I. Corke. A tutorial on visual servo control. IEEE Transactions on Robotics and Automation, 12(5):651670, October 1996

[12] J. Kosecka. Visually guided navigation. In Proc. 4th Int. Symp. on Intelligent Robotic Systems (SIRS'96), pages 77-95, Lisbon, Portugal, July 1996.

[13] A. De Luca, G. Oriolo, and C. Samson. Robot motion planning and control, volume 229 of Lecture Notes in Control and Information Sciences, chapter Feedback control of a nonholonomic carlike robot, pages 171-253. Springer Verlag ISBN 3-540-76219-1, J.P.Laumond Ed, 1998.

[14] Y. Ma, J. Kosecka, and S. S. Sastry. Vision guided navigation for a nonholonomic mobile robot. IEEE Transactions on Robotics and Automation, 15(3):521-37, June 1999.

[15] E. Malis, F. Chaumette, and S. Boudet. 2 1/2 d visual servoing. IEEE Transactions on Robotics and Automation, 15(2):238-250, April 1999.

[16] Y. Mezouar and F. Chaumette. Path planning for robust image-based control. IEEE Transactions on Robotics and Automation, 18(4):534549, August 2002.

[17] Y. Mezouar, H. Hadj Abdelkader, P. Martinet, and F. Chaumette. Visual servoing from $3 \mathrm{~d}$ straight lines with central catadioptric cameras. In Fifth Workshop on Omnidirectional Vision, Omnivis'2004, pages 55-66, Prague, Czech Republic, May 2004.

[18] E. Malis S. Benhimane. Vision-based control with respect to planar and non-planar objects using a zooming camera. In IEEE International Conference on Advanced Robotics, pages 863-869, July 2003.

[19] C. Samson. Control of chained system. application to path following and time-varying stabilization of mobile robot. IEEE Transactions on Automatic Control, 40(1), 1995.

[20] B. Thuilot, C. Cariou, P. Martinet, and M. Berducat. Automatic guidance of a farm tractor relying on a single cp-dgps. Autonomous Robots, 13, 2002.

[21] B. Thuilot, P. Martinet, L. Cordesses, and J. Gallice. Position-based visual servoing: keeping the object in the field of vision. In IEEE International Conference on Robotics and Automation, pages 16241629, Washington DC, USA, May 2002.

[22] D. Tsakiris, P. Rives, and C. Samson. Extending visual servoing techniques to nonholonomic mobile robots. In G. Hager D. Kriegman and A. Morse, editors, The Confluence of Vision and Control, volume 237 of LNCIS, pages 106-117. Springer Verlag, 1998.

[23] K. Usher, P. Ridly, and P. Corke. Visual servoing of a car-like vehicle - an application of omnidirectional vision. Australasian Conference on Robotics an Automation, pages 37-42, Auckland, November 2002.

[24] R. Vidal, O. Shakernia, and S. Sastry. Formation control of nonholonomic mobile robots with omnidirectional visual servoing and motion segmentation. In IEEE International Conference on Robotics and Automation, pages 584-589, Taipei, Taiwan, September 2003.

[25] N. Winter, J. Gaspar, G. Lacey, and J. Santos-Victor. Omnidirectional vision for robot navigation. In Proc. IEEE Workshop on Omnidirectional Vision, OMNIVIS, pages 21-28, South Carolina, USA, June 2000.

[26] Xianghua Ying and Zhanyi Hu. Catadioptric camera calibration using geometric invariants. IEEE Transactions on Pattern Analysis and Machine Intelligence, 26(10):1260-1271, October 2004. 